\title{
Consensus-based recommendations of Australian podiatrists for the prescription of foot orthoses for symptomatic flexible pes planus in adults
}

\author{
Helen A Banwell ${ }^{*}$, Shylie Mackintosh ${ }^{1,3}$, Dominic Thewlis ${ }^{2,3}$ and Karl B Landorf ${ }^{4}$
}

\begin{abstract}
Background: Foot orthoses are commonly used for symptomatic flexible pes planus in adults. However, there are no clinical guidelines for the prescription of customised foot orthoses that are specific to this population. The aim of this study was to investigate prescription habits of Australian podiatrists for customised foot orthoses for symptomatic flexible pes planus in adults and to develop consensus-based practice recommendations for the prescription of these foot orthoses.

Methods: A four round Delphi survey was undertaken with 24 podiatric experts to establish current use and rationale for individual prescription variables of customised foot orthoses for symptomatic flexible pes planus in adults. Round one determined prescription use (consensus) and rounds two, three and four determined the rationale for use (agreement) of prescription variables across the rearfoot, midfoot, forefoot, as well as accommodation and materials used. For consensus and agreement to be accepted, $70 \%$ of the respondents were required to use or agree on the rationale for use of individual prescription variables.

Results: Consensus was reached in round one for two variables, choice of shell material (polyolefin) and when to prescribe a forefoot post balanced to perpendicular. In rounds two, three and four, agreement was reached for 52 statements related to the rationale for use of individual prescription variables, including when to prescribe: an inverted cast pour [heel in an inverted position], an inverted rearfoot post, a medial heel (Kirby) skive, minimal/ maximum arch fill, a medial flange, a forefoot post and common orthotic accommodations.

Conclusion: This study found consensus or agreement for the use of several prescription variables for customised foot orthoses for symptomatic flexible pes planus in adults. The findings were used to develop the Foot orthosis Prescription Recommendations for symptOmatic flexible Pes planus in adults (FootPROP) proforma, to guide clinicians and researchers in the prescription of customised foot orthoses for this population.
\end{abstract}

Keywords: Foot orthoses, Delphi study, Consensus, Pes planus, FootPROP

\section{Background}

Pes planus (also known as flat or low-arched foot) has been estimated to affect up to $23 \%$ of the adult population [1-5]. The condition is classified as rigid or flexible. Rigid pes planus is defined as a congenital, rigid or spastic deformity of the foot, and flexible pes planus as an acquired joint disorder resulting in a valgus foot deformity [6]. Flexible pes planus may be asymptomatic with

\footnotetext{
* Correspondence: helen.banwell@mymail.unisa.edu.au

'International Centre for Allied Health Evidence (iCAHE), School of Health Sciences, University of South Australia, Adelaide, South Australia 5001 Australia

Full list of author information is available at the end of the article
}

little justification for intervention [7] or symptomatic where pain and/or functional limitations are present [8-10]. Accordingly, symptomatic flexible pes planus is a common reason for people seeking intervention [11].

Foot orthoses (FOs) are the most commonly cited intervention for flexible pes planus [12-14]. FOs are inshoe devices that aim to alleviate symptoms, improve function and prevent injury [13-16]. There are no universally accepted classifications for FOs, although they are often broadly categorised as prefabricated or customised [17-19]. A customised FO is tailored for the individual based on a three-dimensional impression or 
image of the plantar foot with adjustments made to influence foot alignment and position [20]. Individual customisation of FOs potentially offers selective and targeted intervention [21]. However, guidelines for prescribing customised FOs for specific foot types, including symptomatic flexible pes planus, are yet to be established, and the literature that is available offers minimal direction or consistency [22-24].

As a consequence, researchers and clinicians investigating the effect of FOs are left to choose the type or prescription with little guidance. A recent systematic review on the use of FOs for pes planus found that the majority of studies standardised the type or prescription approach across all participants [25]. However, a common criticism of customised FOs used in clinical trials is that they are not appropriately prescribed; the underlying concern being that a standardised prescription may not address individual foot morphology and function in the same manner as an individual prescription [21].

With minimal evidence to base prescriptions of FOs for flexible pes planus in adults on, seeking expert podiatry opinion is appropriate as a first step in developing prescription guidelines. Consensus methods are considered a useful method of dealing with conflicting evidence in the absence of guidelines [26-28]. Therefore, the aim of this study was to investigate prescription habits of Australian podiatrists for customised FOs for symptomatic flexible pes planus in adults and to develop consensus-based recommendations for the prescription of these FOs.

\section{Methods}

This study was a four round Delphi survey. A modified Delphi survey was used where participants opinion was sought in round one, and then responses collated and analysed for existing consensus [29]. Responses not reaching consensus were returned to participants for consideration, comment and ranking for levels of agreement in subsequent rounds (Figure 1). The study was approved by the University of South Australias Human Research Ethics Committee (protocol number 26682). All participants provided written informed consent prior to completing the survey.

\section{Participants}

Twenty four expert podiatrists were recruited as participants for the Delphi survey panel. An expert podiatrist was defined as a registered podiatrist who had been practicing for 10 years or more and satisfied at least one of the following inclusion criteria: held an academic position teaching podiatric biomechanical theory or clinical practice within an Australian podiatry program; held a clinical position where the practice was focused on biomechanical assessment and intervention; or had published research on lower limb biomechanics or FOs within the past five years.

Forty-five potential participants were identified via discussions with university leaders of podiatry programs in Australia, as well as current and recent chairs of the Podiatry Board of Australia and Australian Podiatry Associations within each state of Australia. Participants were randomly selected from this list, which was weighted by population of state or territory (Additional file 1), and invited to participate until 24 experts were enrolled. This number of participants captured a broad representation of experts in the podiatry profession in Australia whilst remaining manageable in terms of panel size. Participants completed a preliminary survey of participant characteristics to ensure eligibility for the study. Participants were asked to keep their involvement confidential and intrapanel communication was anonymous through-out the survey.

\section{Survey format}

The Delphi survey was implemented using SurveyMonkey (SurveyMonkey Inc, Palo Alto, California, USA). Prior to the commencement of the main study the survey was piloted in two stages with seven podiatrists who were not participants, to refine the format and question design.

Round one of the main study included questions relating to common prescription variables for FOs across four sections: rearfoot, midfoot, forefoot, as well as accommodations and materials (Additional file 2). It was considered that terminology related to prescription variables and orthosis manufacturing may vary between the states and territories of Australia, therefore traditional terms were adopted for the length of the study (e.g. the prescription of cast pour was surveyed in preference to newer milling procedures) (Table 1).

The rearfoot section covered pouring and balancing of the negative cast, use of a rearfoot post and a medial heel (Kirby) skive [30]. The midfoot section covered the amount of arch fill plus the use of a medial and lateral flange/flare. The forefoot section covered the use of a forefoot post only. Lastly, the accommodations and materials section covered cast and orthotic accommodations and choice of orthotic material. Participants were asked to estimate their percentage use of these prescription variables and detail when, if ever, each variable would be prescribed for adults with symptomatic flexible pes planus. A final summary question listed eight commonly associated signs of symptomatic flexible pes planus and asked participants to indicate their preference for which type of customised FO (e.g. modified Root device, or inverted (Blake) device (Additional file 2)) they would most frequently prescribe $[18,31,32]$. For example, participants were asked to indicate what type of customised FO would 


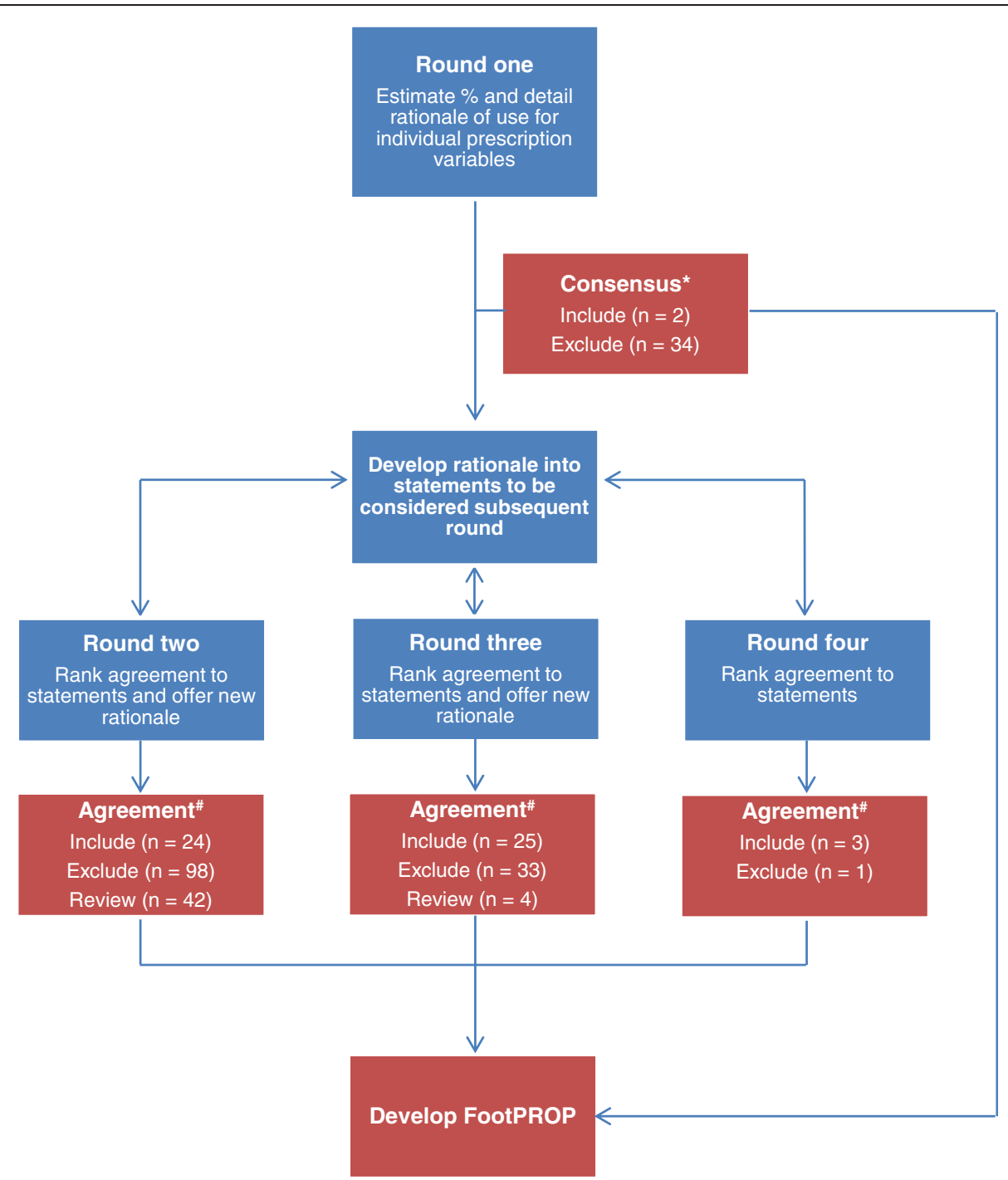

Figure 1 The Delphi survey four round process and results. *Data were considered to have reached consensus if the estimated percent of median use for an individual prescription variable, or the preference for a specified type of FO, was $70 \%$ or more. Data not reaching $70 \%$ were excluded. "Data were considered to have reached agreement if $70 \%$ or more of respondents indicated they agreed or strongly agreed with the statement on a five point Likert scale. Data not reaching 50\% agreement were excluded. Data receiving $50 \quad 69 \%$ agreement were reviewed in subsequent rounds. All data not reaching $70 \%$ agreement at the conclusion of the Delphi were excluded.

be most frequently prescribed in the presence of a moderately lowered navicular height (Additional file 2). In total, round one involved 15 questions relating to 28 individual variables that may be prescribed for adults with symptomatic flexible pes planus and a summary question to determine preference for the type of FO prescribed across eight presentations of flexible pes planus.

Round two was based on the analysis of responses received in round one (Figure 1). Participants comments from round one that related to when, if ever, they would prescribe individual variables were compiled into a list of statements that summarised the full panels rationale for prescription. These statements were distributed to participants in round two. Participants were asked to consider each statement, indicate their level of agreement on a five point Likert scale (i.e. strongly disagree, disagree, neutral, agree or strongly agree), and comment further if desired. As an example, statements related to the prescription of an inverted cast pour [where the cast is poured with the heel bisection in an inverted position] for symptomatic flexible pes planus in adults are displayed in Figure 2. Rounds three and four were developed from responses received during previous rounds in the same manner as round two (Figure 1).

\section{Procedure}

Participants were given the following definition of symptomatic flexible pes planus: 


\section{Table 1 An overview of the individual prescription} variables

\begin{tabular}{ll}
\hline Prescription variable & Method of manufacture \\
\hline Cast Pour & $\begin{array}{l}\text { Negative cast is held in the prescribed } \\
\text { position, based on a bisection of the } \\
\text { posterior heel relative to the supporting } \\
\text { surface, while liquefied plaster is poured } \\
\text { into negative cast }\end{array}$
\end{tabular}

Inverted

Indicates that the negative cast, when poured, is held in an inverted position relative to the heel bisection

Neutral

Everted

Medial heel (Kirby) skive

Indicates that the negative cast, when poured, is held in a vertical position relative to the heel bisection

Indicates that the negative cast, when poured, is held in an everted position relative to the heel bisection

Indicates a small amount of plaster is skived away from the medial heel of the positive cast (skive is generally angled 15 degrees varus/inverted to the plane of the plantar surface of the forefoot post)

Rearfoot post

No post

Extrinsic

Extrinsic (inverted)

Extrinsic (everted)

Extrinsic (with motion)

Arch fill

Minimal

Standard

Maximum

Flange

$\begin{array}{cl}\text { Medial } & \text { A superomedial extension } \\ \text { Lateral } & \text { A superolateral extension } \\ \text { Forefoot post } & \text { A corrective reference platform applied } \\ & \text { to the medial and/or lateral forefoot }\end{array}$

Balanced to perpendicular The reference platform applied to the

Intrinsic plantar forefoot to hold the forefoot alignment as parallel to the supporting surface and perpendicular to the rearfoot

An addition, typically fashioned from a heat mouldable material, that is applied to the final orthosis to stabilise the heel in a vertical position or angle it in the frontal plane (also known as a heel stabiliser)

No external rearfoot post

An external heel post that stabilises the orthosis in a vertical position orthosis into an inverted position orthoses into an everted position

An external heel post that has a biplanar grind on the plantar aspect

The medial longitudinal arch area of the positive cast

A decreased plaster expansion

A standard plaster expansion

An increased plaster expansion

A midfoot extension of the final orthosis border, typically prescribed in conjunction with a deep and distally extended heel cup

The reference platform is applied to the positive cast
Table 1 An overview of the individual prescription variables (Continued)

\begin{tabular}{|c|c|}
\hline Extrinsic & $\begin{array}{l}\text { The platform is applied to the shell of } \\
\text { the final orthosis }\end{array}$ \\
\hline No post & No reference platform applied \\
\hline $1^{\text {st }}$ ray cut out & $\begin{array}{l}\text { The removal of the mediodistal section } \\
\text { (sub } 1^{\text {st }} \text { ray) of the final orthosis }\end{array}$ \\
\hline $1^{\text {st }}$ MTPJ & $\begin{array}{l}\text { The removal of the mediodistal section } \\
\text { (sub } 1^{\text {st }} \text { MTPJ) of the final orthosis }\end{array}$ \\
\hline Plantar fascia groove & $\begin{array}{l}\text { A groove that transverses the long axis } \\
\text { of the orthosis (sub medial slip of the } \\
\text { plantar aponeurosis) }\end{array}$ \\
\hline Metatarsal dome & $\begin{array}{l}\text { A dome-shaped pad applied under or } \\
\text { slightly proximal to the plantar aspect of } \\
\text { the metatarsal heads }\end{array}$ \\
\hline Intrinsic & $\begin{array}{l}\text { The dome is removed from the plaster } \\
\text { of the positive cast }\end{array}$ \\
\hline Extrinsic & $\begin{array}{l}\text { An external dome shaped pad is applied } \\
\text { to the final orthosis }\end{array}$ \\
\hline Cuboid filler & $\begin{array}{l}\text { A increased plaster expansion applied to } \\
\text { sub-calcaneocuboid area of the positive } \\
\text { cast }\end{array}$ \\
\hline Heel aperture & $\begin{array}{l}\text { A circular area removed from the plantar } \\
\text { heel cup of the shell of the final orthosis }\end{array}$ \\
\hline $\begin{array}{l}\text { Notes: MTPJ = metatars } \\
\text { of the foot (generally } \mathrm{m} \\
\text { mould of the foot that } \mathrm{i} \\
\text { negative cast. }\end{array}$ & $\begin{array}{l}\text { joint. Negative cast - plaster cast impression } \\
\text { laster or paris bandage), Positive cast - plaster } \\
\text { s the result of liquefied plaster poured into the }\end{array}$ \\
\hline
\end{tabular}

A deformity that involves pain, fatigue (i.e. painful) or gross biomechanical abnormality (i.e. poorly functioning) that would result in your prescription of foot orthoses during the course of your normal practice. Typical clinical indicators of this foot type are excessive motion around the subtalar joint and rearfoot eversion. This group may also include adultacquired flatfoot (tibialis posterior dysfunction nonfixed deformity) and involve ligamentous laxity, obesity, joint mal-alignment and muscle contractures.

When considering questions and statements, participants were also asked to accept that:

The feet were symptomatic (painful or nonfunctioning); the decision had been made to prescribe custom(ised) foot orthoses; no other pathology existed to influence the prescription choices; and it was limited to the adult client.

Participants were given two weeks to respond to each survey round. Late responders were sent a reminder with a further two weeks extension. Participants were considered non-responders if they failed to complete the survey within one month of the distribution date and had not requested extra time. 


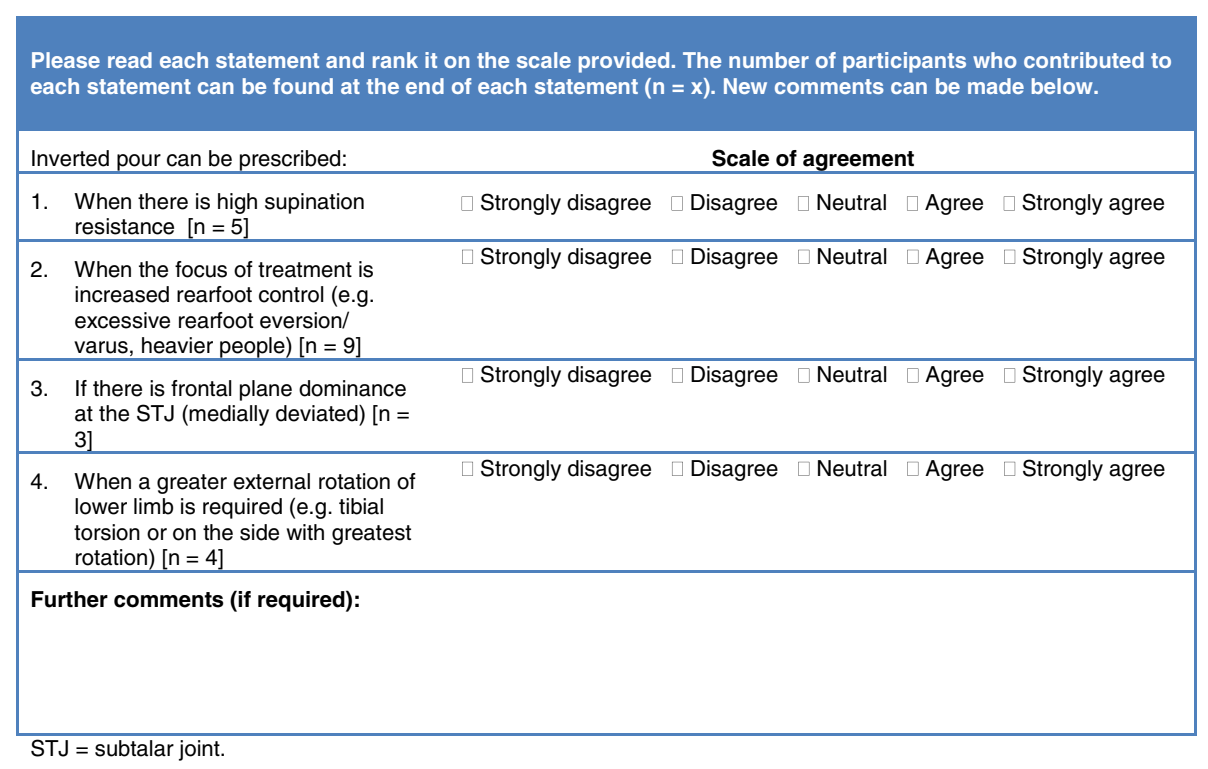

Figure 2 Round two of the Delphi survey on the use of FOs for flexible pes planus in adults. Example taken from the rearfoot section, inverted cast pour [heel in an inverted position].

\section{Data analysis and management}

The outcomes of interest were consensus and agreement on the use of individual prescription variables and the type of customised FO used for symptomatic flexible pes planus in adults. Consensus was based on existing prescription habits and agreement was based on the rationale for the existing prescription habits.

Consensus was determined from round one responses only. It was assessed by: (i) the percentage use of individual prescription variables (Table 2 ), and (ii) the preference for the type of FO that was used for the eight listed presentations of flexible pes planus (Table 3). Consensus was pre-determined at $70 \%$, so for consensus to be accepted the full panel were required to report their combined estimated use or preference as $70 \%$ or more for an individual prescription variable or type of FO respectively. Percentage data for estimated use of individual prescription variables were assessed for normality and found to be skewed; therefore, reported findings are based on group medians (Table 2). Calculation of percentage for the type of FO was based on the number of participants that indicated their preference for each tabled customised orthosis, relative to the number of respondents (Table 3).

Agreement was sought in rounds two, three and four and also determined by percentage, however this was based on the number of participants who agreed with the rationale of a statement relative to the number of respondents to that statement. So, for the use of an individual prescription variable to be accepted, $70 \%$ or more of respondents were required to indicate that they agreed or strongly agreed with the rationale of the statement for the use of that variable (Figure 1). For example, in round one where $100 \%$ of participants responded, at least 17 of the 24 respondents (i.e. $\geq 70 \%$ ) were required to indicate they agreed or strongly agreed with the statement for it to be accepted. Statements where less than $50 \%$ of the respondents agreed were excluded. Statements receiving between 50 to $69 \%$ agreement were reviewed in the following round to ensure adequate consideration from the panel (Figure 1).

Comments provided by participants in a previous round were collated, themed and paraphrased into statements through discussion and agreement of all four authors. The resulting statements were provided to the participants in the next round of the survey with their own original responses from the previous round, to ensure they were satisfied with the management of the data, and so they could review their responses in light of the collated responses.

Data obviously not related to the initial prescription of customised FOs for symptomatic flexible pes planus in adults or not reaching $70 \%$ consensus or agreement at the end of the four round Delphi study were excluded (Figure 1). An a priori decision was made that the Delphi survey would conclude when the response rate dropped below $70 \%$, or round four was complete, irrespective of consensus [29]. Descriptive statistics were undertaken in Microsoft Excel 2010 (Microsoft Corp, Redmond Washington, USA).

\section{Results}

\section{Participant characteristics}

The 24 participants were predominantly male (20 male: 4 female) with an average age of 46.3 years (SD 8.7, 
Table 2 Participants estimated use of individual prescription variables for symptomatic flexible pes planus in the adult (outcomes reaching consensus are bolded)

\begin{tabular}{|c|c|c|c|c|}
\hline Section & Prescription variable & Median\% & SD & Range \\
\hline \multirow[t]{11}{*}{ Rearfoot } & Cast Pour & & & \\
\hline & Neutral [heel in a vertical position] & 60.0 & 32.4 & $0-100$ \\
\hline & Inverted [heel in an inverted position] & 50.0 & 31.9 & $0-90$ \\
\hline & Everted [heel in an everted position] & 0.0 & 7.2 & $0-30$ \\
\hline & Medial heel (Kirby) skive & 25.0 & 29.9 & $0-100$ \\
\hline & Post & & & \\
\hline & No post & 10.0 & 25.1 & $0-100$ \\
\hline & Extrinsic & 50.0 & 32.7 & $0-100$ \\
\hline & Extrinsic (inverted) & 20.0 & 28.2 & $0-90$ \\
\hline & Extrinsic (everted) & 0.0 & 4.4 & $0-10$ \\
\hline & Extrinsic (with motion) & 0.0 & 28.4 & $0-100$ \\
\hline \multirow[t]{7}{*}{ Midfoot } & Arch fill & & & \\
\hline & Minimal & 40.0 & 35.8 & $0-100$ \\
\hline & Standard & 50.0 & 28.5 & $0-90$ \\
\hline & Maximum & 5.0 & 16.3 & $0-50$ \\
\hline & Flange & & & \\
\hline & Medial & 10.0 & 28.9 & $0-100$ \\
\hline & Lateral & 0.0 & 7.2 & $0-30$ \\
\hline \multirow[t]{5}{*}{ Forefoot } & Post & & & \\
\hline & No post & 22.3 & 28.4 & $0-90$ \\
\hline & Intrinsic & 43.6 & 39.2 & $0-100$ \\
\hline & Extrinsic & 11.0 & 11.6 & $0-50$ \\
\hline & Balanced to perpendicular & 50.4 & 40.8 & $0-100$ \\
\hline \multirow[t]{12}{*}{ Accommodations and materials } & $1^{\text {st }}$ ray cut out & 13.1 & 16.5 & $0-60$ \\
\hline & $1^{\text {st }}$ MTPJ cut out & 15.3 & 21.0 & $0-100$ \\
\hline & Plantar fascia groove & 25.0 & 31.4 & $0-100$ \\
\hline & Metatarsal dome & & & \\
\hline & Intrinsic & 5.0 & 28.6 & $0-100$ \\
\hline & Extrinsic & 20.0 & 21.3 & $0-80$ \\
\hline & Cuboid filler & 10.0 & 28.7 & $0-100$ \\
\hline & Heel aperture & 0.0 & 14.9 & $0-50$ \\
\hline & Polyolyenes (e.g. polypropylene) & 75.0 & 31.3 & $0-100$ \\
\hline & Cellular foam (e.g. EVA) & 10.0 & 18.2 & $0-70$ \\
\hline & Composite (e.g. carbon graphite) & 10.0 & 27.1 & $0-98$ \\
\hline & Other & 0.0 & 4.2 & $0-20$ \\
\hline
\end{tabular}

Notes: MTPJ = metatarsophalangeal joint, EVA = Ethylene-vinyl acetate.

range 35 to 67 years). Participants had been practicing for an average of 22.6 years (SD 7.4, range 12 to 39 years) and 16 either held or were currently working towards a recognised post-graduate qualification. Two thirds of the participants listed more than one employment setting; 79\% identified their primary position as a clinician and $91 \%$ worked in a clinical setting at least in a part-time capacity (Additional file 3 ).

\section{Survey findings \\ Consensus}

Two prescription variables reached consensus in round one. Firstly, polyolefin (e.g. polypropylene) was estimated by the participants to be the most frequently prescribed shell material (75\% consensus) (Table 3 ). Secondly, for the eight listed presentations of flexible pes planus, prescription of a forefoot post that was balanced to perpendicular 
Table 3 Percentage of respondents that indicated preference for the type of customised FO prescribed for each listed presentation of symptomatic flexible pes planus in the adult (outcomes reaching consensus are bolded)

\begin{tabular}{|c|c|c|c|c|c|c|c|c|c|c|c|}
\hline \multirow{2}{*}{$\begin{array}{l}\text { Please indicate your preferred FOs } \\
\text { for the listed presentations of } \\
\text { symptomatic flexible pes planus in } \\
\text { adults (mean\%) }\end{array}$} & \multicolumn{3}{|c|}{$\begin{array}{l}\text { Modified root device } \\
\text { (poured to neutral) }\end{array}$} & \multicolumn{3}{|c|}{$\begin{array}{l}\text { Modified root device } \\
\text { (poured to inverted) }\end{array}$} & \multicolumn{3}{|c|}{$\begin{array}{l}\text { Inverted (Blake or } \\
\text { DC wedge) device }\end{array}$} & \multicolumn{2}{|c|}{ Forefoot posts } \\
\hline & alone & + skive & Total & alone & + skive & Total & alone & + skive & Total & Balanced* & Other \\
\hline Moderate rearfoot eversion & 37 & 11 & 48 & 26 & 7 & 34 & 11 & 4 & 15 & 90 & 10 \\
\hline Considerable rearfoot eversion & 4 & 18 & 22 & 18 & 14 & 32 & 25 & 14 & 39 & 89 & 11 \\
\hline Moderate talonavicular bulging & 21 & 14 & 35 & 21 & 14 & 35 & 7 & 7 & 14 & 100 & 0 \\
\hline Considerable talonavicular bulging & 14 & 18 & 32 & 4 & 11 & 15 & 25 & 11 & 36 & 100 & 0 \\
\hline Moderately lowered navicular position & 46 & 7 & 53 & 14 & 7 & 21 & 11 & 0 & 11 & 100 & 0 \\
\hline Considerably lowered navicular position & 14 & 14 & 28 & 21 & 14 & 35 & 14 & 7 & 21 & 100 & 0 \\
\hline Rigid forefoot tilt (varus or valgus) & 21 & 18 & 39 & 21 & 4 & 25 & 7 & 4 & 11 & 79 & 21 \\
\hline Flexible forefoot tilt (varus or valgus) & 33 & 8 & 41 & 17 & 4 & 21 & 17 & 4 & 21 & 81 & 19 \\
\hline
\end{tabular}

Notes: poured to neutral = neutral cast pour [heel in a vertical position], poured to inverted = inverted cast pour [heel in an inverted position], *Balanced = forefoot to be balanced parallel to the supporting surface.

(to the rearfoot) was the most frequently prescribed forefoot option (79 to $100 \%$ consensus) (Table 3). No further consensus was reached.

However, there were several other variables that were frequently prescribed. For the rearfoot, a neutral cast pour [where the cast is poured with the heel bisection in a vertical position] (median use 60\%), and an extrinsic rearfoot post (median use 50\%), were frequently prescribed variables (Table 3). For the midfoot, a standard arch fill (median use 50\%) was the most frequently prescribed variable (Table 2). For the forefoot and accommodation sections, a forefoot post that balanced the forefoot to perpendicular (median use 50\%) and a plantar fascia groove (median use 25\%) were the most frequently prescribed variables respectively (Table 2). In addition, a modified Root-style device (poured to neutral [heel in a vertical position]), with or without a medial heel (Kirby) skive, was the most frequently prescribed type of FO in the presence of moderate rearfoot eversion (48\%), a moderately lowered navicular position (53\%), a rigid forefoot varus or valgus (39\%), or a flexible forefoot varus or valgus (41\%) (Table 3). Both this type of FO and a modified Root-style device (poured inverted [heel in an inverted position]), with or without a medial heel (Kirby) skive, were equally estimated to be the most frequently prescribed in the presence of a moderate talonavicular bulge (35\% respectively). A modified Root-style device (poured inverted [heel in an inverted position]) was also estimated to be the most frequently prescribed in the presence of a considerably lowered navicular position (35\%). An inverted (Blake) style device, with or without a medial heel (Kirby) skive, was the most frequently prescribed in the presence of considerable rearfoot eversion or considerable talonavicular bulge (39 and 36\% respectively) (Table 3).

\section{Agreement}

Round one resulted in the development of 164 statements to be considered in round two (Table 4). After round two, 24 statements were accepted, 98 statements were excluded and 42 statements required review. In addition, 21 new comments were developed in 20 statements to be considered in round three (Table 4). After round three, 25 statements were accepted, 33 statements were excluded and 4 statements required review (Table 4). No new comments were received in round three. After round four, three statements were accepted and one statement excluded (Table 4).

For the rearfoot section, 18 of the 66 statements were accepted (Table 5). Participants agreed that a neutral cast pour [heel in a vertical position] may be prescribed if it reflects the foot position and adequately addresses rearfoot control (Table 5). However, an inverted cast pour [heel in an inverted position], the addition of a medial heel (Kirby) skive, or an extrinsic rearfoot post (inverted) can be prescribed if there is a medially deviated subtalar joint axis [33,34], an increased requirement for rearfoot control, tibialis posterior dysfunction (adult-acquired flatfoot) or a high supination resistance $[35,36]$ (Table 5). There was also agreement from the participants that a medial heel (Kirby) skive may be prescribed when there are concerns

Table 4 Results per round for statements of agreement
considered by participants
\begin{tabular}{lllll}
\hline Round & $\begin{array}{l}\text { Response } \\
\text { rate\% }\end{array}$ & $\begin{array}{l}\text { Statements } \\
\text { accepted (n) }\end{array}$ & $\begin{array}{l}\text { Statements not } \\
\text { accepted (n) }\end{array}$ & $\begin{array}{l}\text { Statements } \\
\text { generated (n) }\end{array}$ \\
\hline 1 & 100 & NA & NA & 164 \\
2 & 100 & 24 & 98 & 20 \\
3 & 91 & 25 & 33 & 0 \\
4 & 91 & 3 & 1 & NA \\
\hline
\end{tabular}

Notes: NA = not applicable. 
Table 5 All accepted statements from the Delphi survey on prescription of customised FOs for symptomatic flexible pes planus in the adult

\section{Inverted cast pour [heel in an}

In the prescription of FOs for symptomatic pes planus, the following can be prescribed when inverted position]

Neutral cast pour [heel in a vertical position]

Medial heel (Kirby) skive

\author{
No rearfoot post \\ Extrinsic rearfoot post \\ Extrinsic rearfoot post (inverted)
}

Minimal arch fil

Maximum arch fill

Medial flange

No forefoot post

Intrinsic forefoot post

Extrinsic forefoot post

Forefoot post balanced to perpendicular

\section{$1^{\text {st }}$ MTPJ cut out}

Plantar fascial groove

There is tibialis posterior dysfunction

There is a high supination resistance

To reflect the foot position accurately

When inversion cannot be tolerated

When there is medial deviation of the STJ axis

When additional rearfoot control required

With tibialis posterior dysfunction

With high supination resistance

Allows increased control without bulk

Greater anti-pronation force required at sustentaculum tali inversion in frontal plane

The focus of treatment is increased rearfoot control (e.g. excessive rearfoot
eversion/varus)

The STJ is medially deviated (frontal plane dominance) $\quad 70.8$

\section{Agreement (\%) Round}

accepted

$$
79.1
$$

$$
81.8
$$

75.0

When rearfoot control is adequately addressed in this position

91.7

$$
91.6
$$

$$
75.0
$$

$$
86.3
$$

To increase the calcaneal inclination in the sagittal plane as well as some

When footwear accommodation is a concern 75.0

2
To increase stability of device

To increase rearfoot control (medially deviated STJ, high supination resistance)

To achieve full amount of correction (when foot ROM allows)

In the presence of range of motion limitations

When there is a severe flat foot deformity (e.g. weight bearing medial cuneiform)

In the presence of large midfoot transverse ROM (talus and/or navicular)

With tibialis posterior dysfunction

When increased medial control is required (midfoot support)

In the presence of forefoot supinatus when the supinatus can be reduced

In the presence of forefoot valgus

To balance forefoot to rearfoot misalignment

When the inverted rearfoot position offers sufficient support to the symptomatic pes planus foot

With severe forefoot supinatus or osseous varus

Standard practice outside of fixed forefoot deformities

To encou
stability)

Maintains rearfoot to forefoot balance

2

When the plantar fascia is tight

When the plantar fascia is prominent (bowstrings)

When the plantar fascia is irritated or painful

To minimise risk of irritation

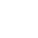

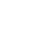

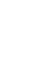


Table 5 All accepted statements from the Delphi survey on prescription of customised FOs for symptomatic flexible pes planus in the adult (Continued)

\begin{tabular}{|c|c|c|c|}
\hline \multirow[t]{3}{*}{ Metatarsal dome } & When forefoot pain exists (e.g. neuroma, bursitis, hyperkeratosis, metatarsalgia) & 87.5 & 2 \\
\hline & In the presence of digital deformities (claw/hammer toes) & 83.4 & 2 \\
\hline & If previously had success with a metatarsal dome & 81.8 & 3 \\
\hline Cuboid filler & Symptomatic lateral column or midfoot (e.g. subluxed cuboid) & 90.9 & 3 \\
\hline Heel aperture & In the presence of plantar calcaneal bursitis & 79.0 & 4 \\
\hline \multirow[t]{8}{*}{ A rigid, semi-rigid and flexible device } & Patient weight/size (increased weight = increased rigidity required) & 91.7 & 2 \\
\hline & Degree of control required (increased control = increased rigidity required) & 87.5 & 2 \\
\hline & Activity levels (increased activity = increased rigidity required) & 77.3 & 3 \\
\hline & Perceived tolerance of patient to rigidity & 90.9 & 3 \\
\hline & Footwear limitations & 72.7 & 3 \\
\hline & Available ROM/joint integrity & 81.8 & \\
\hline & Longevity required from device & 72.7 & 3 \\
\hline & Stability is gained with maximum rigidity & 72.7 & \\
\hline
\end{tabular}

Notes: $\mathrm{STJ}=$ subtalar joint, $\mathrm{ROM}$ = range of motion, $\mathrm{MTPJ}=$ metatarsophalangeal joint.

about device bulk or when greater anti-pronation force is required (Table 5). Furthermore, participants agreed that stability of the device is maximised by an extrinsic rearfoot post, whereas no additional rearfoot post may be prescribed when footwear accommodation is a concern (Table 5). There was no agreement on clinical indicators for the prescription of an extrinsic rearfoot post (everted) or a rearfoot post with motion (Additional file 4).

For the midfoot section, 7 of the 34 statements were accepted (Table 5). The participants agreed a minimum arch fill ensures that the captured foot posture is maintained, which achieves full support (or correction). In contrast, a maximum arch fill may be prescribed in the presence of range of motion limitations or severe flatfoot deformity (Table 5). A medial flange may be prescribed if there is a large amount of transverse plane midfoot motion, tibialis posterior dysfunction (adult-acquired flatfoot) or if increased medial midfoot control is required (Table 5). There was no agreement on clinical indicators for the prescription of a standard arch fill or a lateral flange (Additional file 4).

For the forefoot section, 9 of the 25 statements were accepted (Table 5). Participants agreed that, no forefoot post may be prescribed in the presence of a reducible forefoot supinatus, a soft tissue or positional varus of the forefoot relative to the rearfoot [18], whereas an extrinsic forefoot post may be prescribed in the presence of severe midfoot collapse or a fixed inverted forefoot deformity (Table 5). An intrinsic forefoot post may be prescribed for a severe forefoot supinatus or (osseous) varus, or when the foot exhibits a forefoot valgus, a forefoot to rearfoot misalignment that can be balanced, or when inverting the rearfoot position offers sufficient support (Table 5). However, participants also agreed that outside of fixed forefoot deformities (e.g. reducible forefoot supinatus), balancing the forefoot to perpendicular is considered standard practice as it maintains the rearfoot to forefoot balance and encourages the forefoot to be parallel with the supporting surface (Table 5).

For the accommodations and materials section, 18 of 59 statements were accepted (Table 5). Participants agreed that the use of a $1^{\text {st }}$ metatarsophalangeal joint (MTPJ) cut out may be used in the presence of a plantar-flexed $1^{\text {st }}$ ray and a metatarsal dome may be prescribed for forefoot pain (e.g. sub-metatarsal pain), digital deformities (e.g. flexible clawed toes) or if a metatarsal dome has been used with success previously (Table 5). A plantar fascial groove may be prescribed for a tight, prominent or symptomatic plantar fascia and a cuboid filler prescribed for a symptomatic lateral column or midfoot (Table 5). Participants also agreed that a heel aperture may assist with plantar calcaneal bursitis (Table 5). In relation to materials, a more rigid material can be used when a person is heavier, has increased activity levels, has a foot that requires increased support (i.e. control), or if the FO needs to be more stable or longer lasting. In contrast, reduced thickness and rigidity of the shell material may be chosen if there are tolerance issues, footwear limitations or reduced joint range of motion (Table 5). There was no agreement on clinical indicators that would result in the prescription of a $1^{\text {st }}$ ray cut out (Additional file 4).

\section{Development of results into consensus-based recommendations}

All included data were pooled and delineated into four sections; rearfoot, midfoot, forefoot, and accommodations and materials. The consensus point that polyolefin (e.g. polypropylene) be used as a base material was considered an overall recommendation (http://www.itek.com.au/news-resources/ publications/item/footprop.html). However, the second 
consensus point, that a forefoot post be balanced to perpendicular was considered in light of two conflicting accepted statements. The first of these was that no forefoot post may be prescribed in the presence of forefoot supinatus if the supinatus can be reduced (i.e. there is sufficient motion available for it to function parallel with the supporting surface during weightbearing) (Table 5). The second was that an extrinsic forefoot post could be prescribed when there was a fixed inverted forefoot deformity or severe midfoot collapse (Table 5). Therefore, the recommendation was developed that; other than a reducible forefoot supinatus, a fixed inverted forefoot deformity or severe midfoot collapse, the forefoot post should be intrinsic and balanced to perpendicular to the rearfoot (http://www.itek.com.au/ news-resources/publications/item/footprop.html).

It was also noted that all the accepted statements on the prescription of an inverted cast pour [heel in an inverted position] were consistent with those accepted for the use of a medial heel (Kirby) skive (Table 5). That is, it was accepted that both variables may be prescribed when increased rearfoot control is required or in the presence of a medially deviated subtalar joint axis, tibialis posterior dysfunction (adult-acquired flatfoot) or a high supination resistance (Table 5). This suggests that these two prescription variables are interchangeable, based on practitioner preference or determined by means not captured by this study. Given that both variables reached the inclusion criteria and no evidence exists to guide the choice of one variable over another, the recommendations include both prescription variables with the decision to be at the discretion of the practitioner (http://www.itek.com.au/news-resources/publications/ item/footprop.html).

Following analysis of the findings from this Delphi survey, the consensus variables and the accepted statements were developed into a proforma, the consensus-based Foot orthosis Prescription Recommendations for symptOmatic flexible Pes planus in adults (FootPROP), which can be modified to co-exist with current foot orthosis prescription forms (http://www.itek.com.au/news-resources/ publications/item/footprop.html).

\section{Discussion}

This study has resulted in the first consensus-based recommendations for the prescription of customised FOs for symptomatic flexible pes planus in adults. Both clinicians and researchers may use these recommendations to guide their practice and research. Although the findings of this study suggest that existing prescription habits of expert podiatrists for this condition are not universal, they do indicate that there is agreement on the rationale for use of individual prescription variables (Table 5).

The only previous study relating to practitioner prescribing of foot orthoses by Landorf et al. surveyed Australian and New Zealand podiatrists on their prescription habits in 2001 [37]. They reported that the estimated use of a modified Root-style device was just over half (52\%) of customised FO prescriptions. The findings from our study confirm that the prescription variables common to a standard modified Root-style device are still the most frequently prescribed; that is, a neutral cast pour [heel in a vertical position], a standard arch fill and a forefoot post balanced to perpendicular (Table 2). The standard modified Root-style device was also the most frequently reported preference for five of the eight listed presentations of symptomatic flexible pes planus in adults (4 to 46\%) (Table 3). However, these results demonstrate that a standard modified Root-style device is not universally prescribed for symptomatic flexible pes planus in adults, with variations based on several identified rationales. From participants responses, the aim of prescription for this population is to: (i) influence the level of control of the rearfoot and midfoot with cast pour, rearfoot post, medial heel (Kirby) skive, arch fill and rigidity of the shell choices, (ii) influence or support the forefoot position with forefoot post choices, and (iii) accommodate or minimise painful pathologies and deformities with the accommodation choices.

The findings of our study show that when increased control of the rearfoot is required, prescription of an inverted cast pour [heel in an inverted position], a medial heel (Kirby) skive or an extrinsic rearfoot post (inverted) may be employed (Table 5). The rationale for these prescription options have been previously discussed in the literature [18,30,38-41]. The findings of our study suggest that these prescription variables may be employed interchangeably or as adjunctive options, with the use of an inverted cast pour [heel in an inverted position], specifically, estimated to be prescribed in $50 \%$ of symptomatic flexible pes planus presentations (Table 2).

For the midfoot, increased control may be achieved with the prescription of a minimal arch fill and/or the use of a medial flange (Table 5). Both prescription variables are purported to reduce excessive pronation and assist re-supination of the foot during the mid-stance and propulsive (terminal) phases of gait [40], although there is currently no evidence to support this. The participants in our study estimated that $40 \%$ of prescriptions used a minimal arch fill, again making this a frequently prescribed variation on a standard FO (Table 2).

For the forefoot, the rationale for accepted prescription variables related to the existing forefoot to rearfoot alignment and was dependent on available forefoot motion (Table 5). For example, if there was a reducible forefoot supinatus, it was accepted that balancing the forefoot to perpendicular encourages forefoot alignment to be parallel to the supporting surface (Table 5). However, in the presence of forefoot motion 
limitations, the forefoot may be targeted for support with an extrinsic forefoot post (Table 5), or held in its existing alignment by the prescription of no forefoot post (Table 5). This is in keeping with the literature, which suggests that the aim of extrinsic forefoot posting is to minimise the compensatory effects of a large non-reducible forefoot misalignment [42], whereas when the existing forefoot position is perpendicular to the rearfoot in the frontal plane, capturing this existing aligned position offers sufficient support.

For accommodations, the rationale for prescription appeared primarily based on the presence of existing pathologies (Table 5). Participants agreed that the aim was to accommodate painful conditions (e.g. the use of a plantar fascial groove in the presence of an irritated or painful plantar fascia) or to offload painful plantar metatarsal head regions (e.g. with a metatarsal dome) (Table 5). The recommendations for the use of a semirigid shell material (i.e. polyolefin), appeared targeted at ensuring the FO offers sufficient control of the flexible foot type (Table 5), with the choice of final rigidity guided by perceived client tolerance, footwear concerns and joint integrity or range of motion limitations (Table 5).

Alternative published prescription recommendations for flexible pes planus in adults are sparse. Nonetheless, some similarities exist between the available literature and the outcomes reported in our study. For example, Scherer suggested that in the presence of tibialis posterior dysfunction (adult-acquired flatfoot), a neutral cast pour [heel in a vertical position] with the addition of a medial flange and a medial heel (Kirby) skive be prescribed [23], which is consistent with our findings. Interestingly, Scherer does not advocate specific prescription recommendations for other presentations of flexible pes planus in the adult, only for tibialis posterior dysfunction. Similarly, Rosenbloom recommended a neutral cast pour [heel in a vertical position] with a medial flange, but also recommended an extrinsic rearfoot and forefoot post, with this recommendation aimed at all presentations of flexible pes planus [24]. The findings of our Delphi survey have established that standardising the prescription for flexible pes planus presentations may not reflect existing clinical practice in Australia. Within the limits of this study, this supports the perception that standardising the FOs used in research may not mirror current clinical practice. It is important to note, however, that this issue relates to generalisability of the orthotic prescription, and the authors cannot make any conclusions as to whether this influences the effectiveness of FOs used in either setting. There is no evidence to suggest that one approach to FOs prescription is more, or less, effective than another.

The need to investigate the effectiveness of individualised FOs for adults with symptomatic pes planus is clear. The development of the FootPROP proforma will allow researchers to prescribe customised FOs that are specific for individual signs and symptoms of each study participant. It also offers clinicians the opportunity to review consensus-based recommendations in relation to their own practice methods. The FootPROP proforma requires further evaluation, however it is the first consensus-based prescription recommendations for this specific population and it is envisaged that it will allow for more generalisable research outcomes in the future.

Our findings need to be viewed in the context of several limitations. Firstly, there is no universally accepted definition or classification for flexible pes planus, or consensus on what or when intervention may be required. Secondly, although our findings are a good starting point, they are based on expert opinion, which in the context of evidence-based practice constitutes low-level evidence. In particular, the findings outlined in this study do not constitute evidence that the prescription recommendations lead to an effective FO. Further evaluation with studies that provide higher level evidence (e.g. randomised trials) are needed. Thirdly, the Delphi survey process has been subject to concerns, with the existence of consensus or agreement not necessarily confirming that the answer is correct, as there is a danger of deriving collective ignorance rather than wisdom [26]. Furthermore, anonymity and confidentiality are suggested requirements of participants in Delphi surveys as collusion may affect results. However, given the general collegiate relationships that exist within the Australian podiatric profession, it cannot be guaranteed that participants did not deduce who their fellow survey participants were. Finally, the term expert and its application to health practitioners is controversial [27] and no classification exists within the podiatry profession for expert status. The criteria set for expert within this study were based on discussions between authors and criteria set in other Delphi panels. Accordingly, the findings of this study may not be generalisable to the wider population of podiatric practitioners. This issue extends to podiatrists from other countries, where differences in prescription habits for FOs for adults with flexible pes planus may exist.

\section{Conclusion}

This study found some consistency in FO prescription habits for symptomatic flexible pes planus in adults and that the prescription of individual variables for this condition is based on the following four key rationales. Firstly, when the required foot position is adequately supported in a neutral position, then a standardised modified Root style device (i.e. a neutral cast pour [heel in a vertical position], a standard arch fill and a forefoot post that is balanced to perpendicular) may offer adequate control and support. However, if the aim of the 
intervention is to gain increased control, then the use of an inverted cast pour [heel in an inverted position], an extrinsic rearfoot post (inverted), a medial heel (Kirby) skive, minimal arch fill and/or or a medial flange are modifications that can be incorporated. Secondly, the choice of a forefoot post can be directed by the existing forefoot position. It may be: held in its existing position (no forefoot post), influenced to align the forefoot with the rearfoot when motion allows (an intrinsic balanced forefoot post), or supported in the presence of motion limitation (an extrinsic forefoot post). Thirdly, prescribing orthotic accommodations can be directed by the presence of painful pathologies or digital deformities. Fourthly, the use of polyolefin (e.g. polypropylene) as a base material is recommended, with the final rigidity guided by client characteristics and perceived requirements. Finally, the FootPROP proforma, developed as a result of these findings, will allow investigators evaluating the effectiveness of FOs for flexible pes planus to adopt prescriptions that are more reflective of clinical practice.

\section{Additional files}

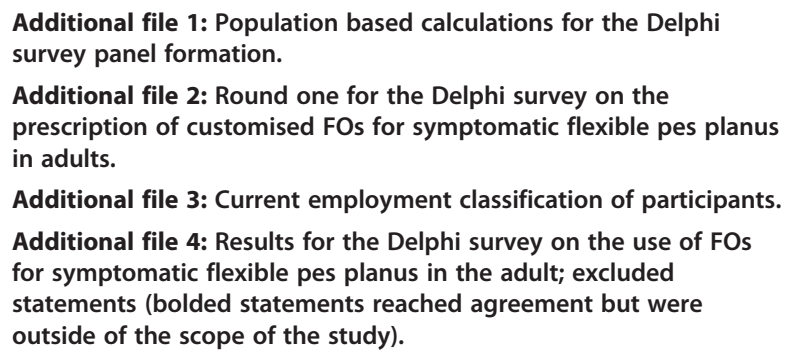

Additional file 3: Current employment classification of participants. Additional file 4: Results for the Delphi survey on the use of FOs for symptomatic flexible pes planus in the adult; excluded statements (bolded statements reached agreement but were outside of the scope of the study).

\section{Competing interests}

KBL is Deputy Editor-in-Chief of the Journal of Foot and Ankle Research it is journal policy that editors are removed from the peer review and editorial decision making process for papers they have co-authored.

\section{Authors contributions}

Initial development of the survey was undertaken by HB with all authors contributing to the final survey. Analyses and management of the data were undertaken by $\mathrm{HB}$ and $\mathrm{DT} / \mathrm{SM} / \mathrm{KL}$ with all four authors being in agreement with the final presented findings. All authors contributed to and approved the final manuscript.

\section{Acknowledgements}

The authors would like to thank those who were involved with piloting the Delphi survey and sincerely acknowledge the generous contribution the expert panel provided towards this research. HB was funded by an Australian Postgraduate Awards scholarship during the course of this study.

\section{Author details}

'International Centre for Allied Health Evidence (iCAHE), School of Health Sciences, University of South Australia, Adelaide, South Australia 5001, Australia. ${ }^{2}$ Biomechanics and Neuromotor Laboratory, School of Health Sciences, University of South Australia, Adelaide, South Australia 5001, Australia. ${ }^{3}$ Sansom Institute for Health Research, University of South Australia, Adelaide, South Australia 5001, Australia. ${ }^{4}$ Department of Podiatry and Lower Extremity and Gait Studies Program, Faculty of Health Sciences, La Trobe University, Victoria 3083, Australia.
Received: 22 August 2014 Accepted: 5 November 2014

Published online: 25 November 2014

\section{References}

1. Dunn J, Link C, Felson D, Crincoli M, Keysor J, McKinlay J: Prevalence of foot and ankle conditions in a multiethnic community sample of older adults. Am J Epidemiol 2004, 159:491 498

2. Garrow A, Silman A, Macfarlane G: The Cheshire Foot Pain and Disability Survey: a population survey assessing prevalence and associations. Pain 2004, 110:378 384.

3. Kosashvili Y, Fridman T, Backstein D, Safir O, Bar Ziv Y: The correlation between pes planus and anterior knee or intermittent low back pain. Foot Ankle Int 2008, 29:910 913

4. Golightly YM, Hannan MT, Dufour AB, Jordan JM: Racial differences in foot disorders and foot type. Arthritis Care Res (Hoboken) 2012, 64:1756 1759

5. Shibuya N, Jupiter DC, Ciliberti LJ, VanBuren V, La Fontaine J: Characteristics of adult flatfoot in the United States. Foot Ankle Surg 2010, 49:363 368 .

6. WHO: ICD-10 Diseases of the musculoskeletal system and connective tissue. Geneva: World Health Organisation; 2010.

7. Staheli L: Planovalgus foot deformity. Current status. J Am Podiatr Med Assoc 1999, 89:94 99.

8. Evans A: The flat-footed child - to treat or not to treat. What is the clinician to do? J Am Podiatr Med Assoc 2009, 99:179.

9. Sullivan J: Pediatric flatfoot: evaluation and management. J Am Acad Orthop Surg 1999, 7:44 53.

10. Wilson MJ: Pes Planus. A synopsis of causation. United Kingdom: Ministry of Defence; 2008

11. Bennett PJ: Types of foot problems seen by Australian podiatrists. Foot 2012, 22:40 45.

12. Esterman A, Pilotto $L$ : Foot shape and its effect on functioning in Royal Australian Air Force recruits. Part 1: Prospective cohort study. Mil Med 2005, 170:623 628 .

13. Hume P, Hopkins W, Rome K, Maulder P, Coyle G, Nigg B: Effectiveness of foot orthoses for treatment and prevention of lower limb injuries: a review. Sports Med 2008, 38:759.

14. Collins N, Bisset L, McPoil T, Vicenzino B: Foot orthoses in lower limb overuse conditions: a systematic review and meta-analysis. Foot Ankle Int 2007, 28:396 410.

15. Landorf K, Keenan A-M: Efficacy of foot orthoses - what does the literature tell us? J Am Podiatr Med Assoc 2000, 90:149 158.

16. Landorf KB, Keenan A-M: Do foot orthoses prevent injury. In Evidence-based sports medicine. 2nd edition. Edited by MacAuley D, Best TM. Malden, Mass: Blackwell Pub; 2007:73 92.

17. Australian Podiatry Council: Clinical guidelines for orthotic therapy provided by Podiatrists. In Australian Podiatry Manual Supplement. Collingwood Victoria: Australian Podiatry Council; 1998:18.

18. Valmassy RL: Clinical biomechanics of the lower extremities. St. Louis: Mosby; 1996.

19. Michaud TC: Human Locomotion: The Conservative Management of Gait-Related Disorders. Newton, Mass: Newton Biomechanics; 2011

20. Berenter R, Kosai D: Various types of orthoses used in podiatry. Clin Podiatr Med Surg 1994, 11:219 229.

21. Menz H: Foot orthoses: how much customisation is necessary? J Foot Ankle Res 2009, 2:23.

22. ACFAOM: Prescription Custom Foot Orthoses Practice Guidelines. Bethesda, MD: The American College of Foot and Ankle Orthopaedics and Medicine; 2011.

23. Scherer P: Recent Advances in Orthotic Therapy. Improving clinical outcomes with a pathology-specific approach. New York: Lower Extremity Review, LLC: 2011.

24. Rosenbloom KB: Pathology-designed custom molded foot orthoses. Clin Podiatr Med Surg 2011, 28:171 187.

25. Banwell H, Mackintosh S, Thewlis D: Foot orthoses for adults with flexible pes planus: a systematic review. J Foot Ankle Res 2014, 7:23.

26. Jones J, Hunter D: Consensus methods for medical and health services research. Br Med J 1995, 311:376 380.

27. Hasson F, Keeney S, McKenna H: Research guidelines for the Delphi survey technique. J Adv Nurs 2000, 32:1008 1015.

28. Sinha IP, Smyth RL, Williamson PR: Using the Delphi technique to determine which outcomes to measure in clinical trials: 
recommendations for the future based on a systematic review of existing studies. PLoS Med 2011, 8:e1000393.

29. Vernon W: The Delphi technique: a review. Int J Ther Rehabil 2009, 16:69 76.

30. Kirby $\mathrm{K}$ : The medial heel skive technique, Improving pronation control in foot orthoses. J Am Podiatr Med Assoc 1992, 82:177 188.

31. Root ML: Development of the functional orthosis. Clin Podiatr Med Surg 1994, 11:183 210.

32. Blake RL: Inverted functional orthoses. J Am Podiatr Med Assoc 1986, 76:275 276 .

33. Kirby KA: Methods for determination of positional variations in the subtalar joint axis. J Am Podiatr Med Assoc 1987, 77:228 234.

34. Kirby KA: Subtalar joint axis location and rotational equilibrium theory of foot function. J Am Podiatr Med Assoc 2001, 91:465 488.

35. Kirby KA, Green DR: Evaluation and Nonoperative Management of Pes Valgus, pp. 295 327. In Foot and Ankle Disorders in Children. Edited by DeValentine S. New York: Churchill-Livingstone; 1992.

36. Payne $\mathrm{CB}$, Munteanu S, Miller K: Position of the subtalar joint axis and resistance of the rearfoot to supination. J Am Podiatr Med Assoc 2003, 93:131 135

37. Landorf K, Keenan A, Rushworth R: Foot orthosis prescription habits of Australian and New Zealand podiatric physicians. J Am Podiatr Med Assoc 2001, 91:174 183.

38. Ferguson $\mathrm{H}$, Blake R: Update and rationale for the inverted functional foot orthosis. Clin Podiatr Med Surg 1994, 11:311 337.

39. Hice GA: Orthotic treatment of feet having a high oblique midtarsal joint axis. J Am Podiatr Med Assoc 1984, 74:577 582.

40. Kirby K: Foot and Lower Extremity Biomechanics. A Ten Year Collection of Prescision Intricast, Inc. newsletters. Precision Intricast, Inc: Payson, Arizona; 1997.

41. Williams DS III, McClay Davis I, Baitch SP: Effect of inverted orthoses on lower-extremity mechanics in runners. Med Sci Sports Exerc 2003, 35:2060 2068

42. Pratt DJ: Functional foot orthoses. Foot 1995, 5(3):101 110

doi:10.1186/s13047-014-0049-2

Cite this article as: Banwell et al: Consensus-based recommendations of Australian podiatrists for the prescription of foot orthoses for symptomatic flexible pes planus in adults. Journal of Foot and Ankle Research 2014 7:49.

\section{Submit your next manuscript to BioMed Central and take full advantage of:}

囚Convenient online submission

$\otimes$ Thorough peer review

$\otimes$ No space constraints or color $\nabla$ gure charges

$\triangle$ Immediate publication on acceptance

Q Inclusion in PubMed, CAS, Scopus and Google Scholar

$\otimes$ Research which is freely available for redistribution 